\title{
Calculation of Spare Parts in a Car Service Enterprise Based on Evaluating the Average Time of Details Serviced Automobiles
}

\author{
Karimov Nijat Ashraf \\ Department of Automotive Engineering, Azerbaijan Technical University, Baku, Azerbaijan
}

Email address:

nicat.kerimov12@gmail.com

\section{To cite this article:}

Karimov Nijat Ashraf. Calculation of Spare Parts in a Car Service Enterprise Based on Evaluating the Average Time of Details Serviced Automobiles. International Journal of Science, Technology and Society. Vol. 5, No. 5, 2017, pp. 146-152. doi: 10.11648/j.ijsts.20170505.12

Received: July 11, 2017; Accepted: July 25, 2017; Published: August 15, 2017

\begin{abstract}
The purpose of the work is the development of a technique for calculating spare parts in an auto service company based on an analysis of statistical information on the failure of details of each standard accumulated on daily information on the replacement of spare parts for serviced vehicles in previous planning periods. In the theory of system reliability, the calculation of the failure distribution function usually uses information on the main characteristic of failures - the time between failures, and in the absence of such information, it is necessary to use information on the number of failures at the time of receipt for maintenance of cars in this auto service plant. In this connection, the relationship between the distribution functions of these random variables is established using the reciprocity of the distribution processes to failure and the number of failures. For each of the competing theoretical functions of the distribution of the operating time to failure, an estimate is made of the average operating time of the details of each standard in the auto service plant on the basis of actual demand. The estimates obtained make it possible to calculate the number of spare parts for the subsequent replenishment period of the SPIE.
\end{abstract}

Keywords: Spare Parts Kit SPIA, Operating Time to Failure, Replenishment Period, Diffusion Distribution, The Criterion of Consent

\section{Introduction}

An analysis of previous work on the calculation of spare parts in car service stations (CSS) [1] shows that in recent years, most Russian auto service enterprises have been using the system for determining the need for spare parts, which is based on actual demand and individual details for the previous period Work.

The most important a priori information, which ultimately determines the amount of spare parts, is the theoretical model of failures that is taken into account in calculating the number of failures [2-6].

First of all, on the basis of failure statistics, the best model for the distribution of failures among a finite number of competing theoretical distribution functions is chosen, based on the Kolmogorov-Smirnov agreement, for the first part of each component model, including the diffusion (monotonic and no monotonic) distributions. For the best model for the distribution of failures of parts of each standard, an estimate of the mean time to failure (i.e., the average time between successive replacements) of the details of each standard is calculated taking into account the adequacy index of ensuring non-renewable spare parts [9]. According to these estimates, the expected size of the spare parts of each standard is determined, taken as the initial approximation of the proposed algorithm for calculating spare parts for the subsequent replenishment period SPIE.

It should be noted that the estimation of the mean time to failure of details is calculated from the statistics of their failures. At the same time, the relationship established between the distribution functions of the operating time to failure and the number of failures is substantially used in this paper, the use of which makes it possible to reduce the problem of estimating the mean time to failure to solve a nonlinear algebraic equation. The choice of the best model for the distribution of failures of the part and detail of each 
standard is carried out in accordance with KolmogorovSmirnov's consent criterion. The advantage of this criterion is that it is the only agreement criterion that, along with the choice of the theoretical distribution function, the least deviation from the empirical distribution function in the metric of the space of continuous functions, gives a confidence interval of deviation in the indicated metric.

Obviously, the higher the required level of reliability of providing non-renewable spare parts of a certain type and, correspondingly, the sufficiency index of this level of security, the higher the level of the required number of spare parts in the planned period.

\section{Competing Theoretical Distribution Functions}

(1). Exponential Distribution (E)

$$
F_{T}\left(t ; \mu_{T}\right)=1-\exp \left(-\frac{1}{\mu_{T}}\right)
$$

where $\mu_{T}=1 / \lambda, \lambda$ is the intensive change $T ; \mu_{T}$ is the mathematical expectation of random variable of operating time $T$.

(2). Normal Distribution (N)

$$
F_{T}\left(t ; \mu_{T}, v_{T}\right)=\Phi\left(-\frac{t-\mu_{T}}{\mu_{T} \cdot v_{T}}\right)
$$

where $\Phi(z)$ is standardized normal distribution; $v_{T}=$ $\sqrt{D_{T}} / \mu_{T}$ is the coefficient variation and $D_{T}$ is the dispersion of r.v. $T$.

(3). Logarithmic Normal Distribution $(L N)$

$$
F_{T}\left(t ; \mu_{T}, v_{T}\right)=\Phi\left(\frac{\ln \left[\frac{t\left(1+v_{T}^{2}\right.}{\mu_{T}}\right]}{\left[\ln \left(1+v_{T}^{2}\right)\right]^{1 / 2}}\right)
$$

(4). We Bull Distribution (W)

$$
F_{T}\left(t ; \mu_{T}, v_{T}\right)=1-\exp \left\{-\left[\frac{t \Gamma\left(1+v_{T}\right)}{\mu_{T}}\right]^{1 / v_{T}}\right\}
$$

where $\Gamma(z)$ is gamma function.

(5). Diffusion Non-monotonic Distribution (DN)

$$
F_{T}\left(t ; \mu_{T}, v_{T}\right)=\Phi\left(\frac{t-\mu_{T}}{v_{T} \sqrt{t \cdot \mu_{T}}}\right)+\exp \left(\frac{2}{v_{T}^{2}}\right) \cdot \Phi\left(-\frac{t+\mu_{T}}{v_{T} \sqrt{t \cdot \mu_{T}}}\right)
$$

(6). Diffusion Monotonic Distribution (DM)

$$
F_{T}\left(t ; \mu_{T}, v_{T}\right)=\Phi\left(\frac{t-\mu_{T}}{v_{T} \sqrt{t \cdot \mu_{T}}}\right)
$$

It should be noted that among the competing hypotheses on the form of the distribution function of the operating time to failure, have included the diffusion no monotonic (DN) and diffusion monotonic (DM) distributions related to the probability-physical models representing the new technology of reliability research [10]. In our opinion, diffusion models for the distribution of failures must necessarily be considered among the competing ones, since each element of the system that is malfunctioning and replaced, not immediately after failure, may somehow affect the functioning of other elements (ie, a diffusion of faults from one element occurs point to another). Even the replacement of a malfunctioning element that has had time to influence other elements cannot result in regeneration (ie, complete restoration) of the system.

In Section 5, random variables (r.v) $T_{r_{0}}$ and $R_{r_{0}}$ are taken into consideration for an arbitrary vehicle part, and for a part of the $i$-th type random values of $T_{i, r_{0}}$ and $R_{i, r_{0}}$ are taken into consideration. In this case, $T_{r_{0}}$ is a random variable with values $t, t \leq t_{r_{0}}$, where $t_{r_{0}}=r_{0} \cdot T_{0}, T_{0}, \mathrm{~T}_{0}$ is mathematical expectation of the operating time until failure of any parts; $R_{r_{0}}$ is a random value of failures with values $r, r \leq r_{0}$, $r_{0}=t_{r_{0}} / T_{0}$.

The validity of the equalities is confirmed.

$$
\begin{gathered}
\mu_{T_{r_{0}}}=T_{0} \cdot \mu_{R_{r_{0}}} \\
v_{T_{r_{0}}}=v_{R_{r_{0}}}
\end{gathered}
$$

For any part and equalities, the followings are taken:

$$
\begin{gathered}
\mu_{T_{r_{i, 0}}}=T_{0, i} \cdot \mu_{R_{r_{i, 0}}} \\
v_{T_{r_{i, 0}}}=v_{R_{r_{i, 0}}}
\end{gathered}
$$

for the parts of $i$ type, where $T_{r_{i, 0}}-$ r.v. with values of $t, t \leq t_{r_{i, 0}}, t_{r_{i, 0}}=r_{i, 0} \cdot T_{0, i}, T_{0, i}, \mathrm{~T}_{0, \mathrm{i}}$ is the mathematical expectation of the operating time until failure of the $i$ type part, $R_{r_{i, 0}}-$ r.v. failures with values $r_{i}, r_{i} \leq r_{i, 0}, r_{i, 0}=$ $t_{r_{i, 0}} / T_{0, i}$.

The distribution functions of the quantities $T_{r_{0}}$ and $R_{r_{0}}$ are related by the relation:

$$
F_{T_{r_{0}}}\left(t ; \mu_{T_{r_{0}}}, v_{T_{r_{0}}}\right)=F_{R_{r_{0}}}\left(r ; \mu_{R_{r_{0}}}, v_{R_{r_{0}}}\right),
$$

which allows to pass from the distribution functions $1^{0}-6^{0}$ for the value of $T$ to the distribution functions of the quantity $R$.

Such a connection between the random variables $T_{r_{0}}$ and $R_{r_{0}}$ plays an extremely important role, because, as a rule, the service center has a database on the number of failures (ie replaced) of each type of parts on the basis of cars received for maintenance Days (see, for example, Table 1 below).

As will be shown in Section 3, knowledge of the distribution of the random variable $R_{r_{0}}$ makes it possible not only to determine the distribution of the random variable $T_{r_{0}}$, but also to estimate the mean time to failure (ie, the average time between two successive failures) Which can estimate the number of spare parts of each type of par value for the entire replenishment period $\mathrm{T}_{\mathrm{RP}}$.

The initial data on the failures of parts replaced at the auto service plant under review in the order of arrival of vehicles in each of the 27 working days are shown in Table 1. For the replenishment period $T_{R P}$, one month is taken from 27 working days. 
Table 1. Initial data on the receipt of applications.

\begin{tabular}{llllll}
\hline Days & Engine & Suspension & Body & Total & Number of cars received \\
\hline 1 & 35 & 2 & 2 & 39 & 13 \\
2 & 18 & 3 & 2 & 23 & 8 \\
3 & 33 & 10 & 4 & 47 & 16 \\
4 & 33 & 5 & 5 & 43 & 13 \\
5 & 28 & 5 & 3 & 36 & 17 \\
6 & 28 & 3 & 6 & 37 & 21 \\
7 & 37 & 7 & 3 & 47 & 28 \\
8 & 66 & 3 & 9 & 78 & 15 \\
9 & 26 & 8 & 3 & 37 & 8 \\
10 & 38 & 6 & 4 & 48 & 19 \\
11 & 17 & 9 & 2 & 28 & 38 \\
12 & 32 & 3 & 8 & 43 & 36 \\
13 & 37 & 27 & 3 & 67 & 35 \\
14 & 40 & 8 & 80 & 128 & 11 \\
15 & 48 & 15 & 10 & 73 & 26 \\
16 & 41 & 9 & 77 & 127 & 12 \\
17 & 25 & 13 & 3 & 32 & 25 \\
18 & 25 & 3 & 8 & 46 & 22 \\
19 & 14 & 4 & 1 & 18 & 38 \\
20 & 48 & 7 & 4 & 56 & 13 \\
21 & 37 & 10 & 7 & 51 & 61 \\
22 & 50 & 2 & 7 & 67 & 25 \\
23 & 20 & 3 & 10 & 32 & 31 \\
24 & 14 & 15 & 13 & 140 & \\
25 & 58 & 7 & 71 & 61 & \\
26 & 37 & 13 & 17 & & \\
27 & 34 & & & & \\
\hline
\end{tabular}

On the base of the initial data from table 1 on the failure details $r_{k}\left(k=1, \ldots, n_{0} ; n_{0}=27\right)$ in the $k$ working days of a month, the accumulated amount of failures of parts by the time is calculated $r_{k}^{H}=\sum_{k^{\prime}=1}^{k} r_{k}$ and a step function of continuous time $t$ is introduced on a time scale $t$ with a unit of measurement for one working day, $r^{H}(t)=\sum_{k \leq t} r_{k}$.

The results of the calculations are given in Tables 2 and 3 .

Table 2. The critical values $d_{\alpha}$ of the statistics $D_{n}$.

\begin{tabular}{llll}
\hline & $\boldsymbol{m i n}$ & $\boldsymbol{m a x}$ & $\boldsymbol{\alpha}$ \\
\hline 1 & 0 & 0,294 & 0,05 \\
2 & 0,294 & 0,352 & 0,01 \\
3 & 0,352 & 0,421 & 0,001 \\
4 & 0,421 & 1 & 0 \\
\hline
\end{tabular}

Table 3. Determination of the best failure distribution of any part and the parts of each nominal type.

\begin{tabular}{|c|c|c|c|c|c|c|c|c|}
\hline Method & Engine & & Suspe & & Body & & Total & \\
\hline & $D$ & $\alpha$ & $D$ & $\alpha$ & $D$ & $\alpha$ & $D$ & $\alpha$ \\
\hline 1 & 0,172 & 0,95 & 0,196 & 0,95 & 0,32 & 0,95 & 0,198 & 0,95 \\
\hline 2 & 0,89 & 0 & 0,899 & 0 & 0,99 & 0 & 0,923 & 0 \\
\hline 3 & 4,27 & 0 & 14,3 & 0 & 8,3 & 0 & 4,5 & 0 \\
\hline 4 & 0,675 & 0 & 0,5 & 0 & 0,32 & 0,99 & 0,53 & 0 \\
\hline 5 & 0,16 & 0,95 & 0,182 & 0,95 & 0,29 & 0,95 & 0,172 & 0,95 \\
\hline 6 & 8,9 & 0 & 79,1 & 0 & 47,1 & 0 & 11 & 0 \\
\hline
\end{tabular}

It is seen from table 2 and 3 that, for any part of the best distribution function is $F\left(r^{H}\right)=F_{5}\left(r^{H}\right)$, and for the parts of the nominal type it is $F^{*}\left(r_{i}^{H}\right)=F_{5}\left(r^{H}\right)$, for $i=1,2$, $F\left(r_{i}^{H}\right)=F_{4}\left(r_{i}^{H}\right)$ for $i=3$.

Consequently, in the considered examples diffusion nonmonotonic distribution $(D N)$ is the most concordant with empirical distribution of the failures both in case of any part and for any part of $i=1$, and $i=2$.

\section{Reliability Evaluation of the of Parts and Sufficiency Indicators of SPIA}

The required level of the "reliability" indicator of the products (nodes) at the end of the replenishment period $T_{R P}$ will be established: the probability of failure-free operation the $R_{\text {prod }}^{\text {req }}$, for example, $R_{\text {prod }}^{\text {req }}=0,9$ (this means that at the end of the $T_{R P}$ period there at least $0,1 \cdot N$ details must remain from the planned store of spare parts $(N)$.

The sufficiency indicators of the SPIE $\pi_{S}$ is determined on the base of the analysis of expected $\left(R_{0}\left(T_{R P}\right)\right)$ and required $R_{\text {prod }}^{\text {req }}$ reliability indicators $\left(\pi_{\partial} \geq R_{\text {prod }}^{\text {req }} / R_{0}\left(T_{R P}\right)\right)$ rounding to the nearest values of the series:

$$
0.9 ; 0.95 ; 0.99 ; 0.995 ; 0.999 ; 0.9995 ; 0.9999
$$

It should be noted that the sufficiency index of SPIA $\pi_{s}$ can be set by the customer independently of the expected 
reliability of the product.

To evaluate the sufficiency of single complex of SPIA-S products with non-recoverable spare elements, $\pi_{S}$ is used as the probability so that during the operating time $T_{R P}$, of the product will occur in no failures of SPIA. The probability $\pi_{s}$ is used to estimate the sufficiency of a set of SPIA provided that, all store in this set are replenished periodically with identical periods and the reliability indicators of the product is the probability of failure-free operation. The initial data for calculating the sufficiency index $\pi_{s}$ of the SPIA-S are is the expectal probability of failure-free operation on the final replenishment period of SPIA and the requirements for the reliability index of the product $R_{\text {prod }}^{\text {req }}$.

If it is not known $R_{0}\left(T_{R P}\right)$, then it is calculated

$$
R_{0}\left(T_{R P}\right)=1-F^{*}\left(r\left(T_{R P}\right)\right)
$$

where $F^{*}(r)$ is the most adequate theoretical model of the failures distribution of any type of products, the choice of which is described in section 2. For $\pi_{s}$, the number rounding to the nearest values of the series (11) is adopted. If $R_{\text {prod }}^{\text {req }} / R_{0}\left(T_{R P}\right)=0,9$, then $\pi_{s}:=0,9$.

\section{Evaluation of the Required Probability of Failure-Free Operation of the Elements}

A value of $R_{\text {prod }}^{\text {req }}$ is adopted satisfying the relation $R_{\text {prod }}^{\text {req }} \leq R_{0}\left(T_{R P}\right)$ and according to the value of the series (11), the sufficiency index of a set of SPIE-O is determined by the ratio $\pi_{s} \geq R_{\text {prod }}^{r e q} / R_{0}\left(T_{R P}\right)$ If $R_{0}\left(T_{R P}\right)$ is less than 0.9 , then the value $\pi_{s}=R_{\text {prod }}^{\text {req }}$ is accepted from reasoning on importance of feasible functions and the importance of the economic expediency.

Further, the required probability level of fail-free operation is calculated $R_{i}^{r e q}$ for the elements of the $i$ nominal type $\left(R_{i}^{r e q}=\left(R_{\text {prod }}^{r e q}\right)^{\frac{1}{m}}\right)$, rounding to the nearest value of the series (11), where $m$ is the total number of car's nominal types (nodes) (in our case $m=3$ ).

\section{Probability Evaluation of Fail-Free Operation and Sufficiency of Any Type of Spare Elements}

The calculation of the probability of failure-free operation of $i$ type elements $R_{i}$ at the considered period $\left(0, t_{\Sigma}+T_{R P}\right)$ will be carried out by the formula:

$$
R_{i}=1-F_{i}^{*}\left(r_{i}^{H}\left(t_{\Sigma}+T_{R P}\right)\right)
$$

where $F_{i}^{*}\left(r_{i}^{H}\right)$ is the most adequate theoretical distribution model of the failure of $i$-th type of elements; $t_{\Sigma}$ is the total operating time of the products at the beginning of the replenishment period, taking into account the intensity coefficient at the operation during this period.

According to the values of $R_{i}$ and $R_{i}^{r e q}$, the sufficiency index $\pi_{s}$ for the guarantee of to provide $i$ type nonrecoverable spare parts is determined (rounding to the nearest larger value from the recommended series (11) by the formula (by the condition $R_{i}<R_{i}^{r e q}$ )

$$
\pi_{s i}^{r e q}=\left\{\begin{array}{c}
1-\frac{1-R_{i}^{r e q}}{1-R_{i}}, \text { at } R_{i}<\left(\pi_{s}\right)^{1 / m}, \\
\left(\pi_{s}\right)^{1 / m}, \text { at } R_{i} \geq\left(\pi_{s}\right)^{1 / m}
\end{array}\right.
$$

If $R_{i} \geq R_{i}^{r e q}$, then the elements of this type are n't included in the nomenclature of SPIA (in this case the number of the spare parts $a_{i}$ in the planned period is accepted equal $a_{i}=0$ ); $m$ is the total number of $i$ type (in our case $m=3)$.

\section{The Calculation of Mean Operating Time Until the Failures of Constituent Elements}

Suppose that $T$ is a random variable (abbreviated r.v) of operating time until failure (i.e. the time between two consistent failures) with the values $t$, but $T_{r}$ - r.v. operating time until $r$-th failure (beginning from the initial moment of the time) with the values $t_{r}$. Suppose, further $R$ is r.v. of the failure member with values $\mathrm{r}$ and $R_{r_{0}}$ is r.v. with values $r \leq r_{0}$, where $r_{0}$ is some fixed natural number. Suppose that, $\left\{r_{k}\right\}, k=1, \ldots, n$ is a given sample of observations random variable $R$ and $\left\{t_{k}\right\}$ is the corresponding (unobservable) sequence of a values random variable $T$ with mathematical expectation $\mu_{T}=T_{0}$, where $T_{0}$ is the quantity to be determined.

It is obvious that,

$$
t_{r}=r \cdot T_{0}
$$

consequently,

$$
t_{r_{k}}=r_{k} \cdot T_{0}
$$

Taking into account random variable $T_{r_{0}}$ with values $t \leq t_{r_{0}}$

where

$$
t_{r_{0}}=r_{0} \cdot T_{0}
$$

Suppose $r_{0}=r_{k_{0}}$ is the clement of sequence $\left\{r_{k}\right\}, r_{1}<$ $r_{2}<\cdots<r_{n}$.

The sampling estimation of the mathematical expectation $\mu_{T_{r_{0}}}$ is the quantity.

Suppose $r_{0}=r_{k_{0}}$ is the clement of sequence $\left\{r_{k}\right\}, r_{1}<$ $r_{2}<\cdots<r_{n}$.

The sampling estimation of the mathematical expectation $\mu_{T_{r_{0}}}$ is the quantity.

$$
\hat{\mu}_{T_{r_{0}}}=\sum_{k=1}^{k_{0}} t_{r_{k}}=\sum_{k=1}^{k_{0}} r_{k} \cdot T_{0}=T_{0} \cdot \sum_{k=1}^{k_{0}} r_{k}=T_{0}
$$


consequently,

$$
\mu_{T_{r_{0}}}=T_{0} \cdot \mu_{R_{r_{0}}}
$$

Denote $t_{k}=t_{r_{k}}, t_{0}=t_{r_{0}}=t_{r_{k_{0}}}$, but further the notation $T_{r_{0}}$ and $T_{t_{0}}$ will not be distinguished.

It is obvious that

$$
v_{T_{r_{0}}}=v_{R_{r_{0}}} \text {. }
$$

Taking into account (19)-(20), distribution functions of values $T_{r_{0}} R_{r_{0}}$ is connected with the relation

$$
\begin{gathered}
F_{T_{r_{0}}}\left(t ; \mu_{T_{r_{0}}}, v_{T_{r_{0}}}\right)=\operatorname{Bep}\left(T \leq t \mid r \leq r_{0}\right)= \\
=F_{T_{r_{0}}}\left(t ; \mu_{R_{r_{0}}} \cdot T_{0}, v_{T_{r_{0}}}\right)=F_{R_{r_{0}}}\left(r ; \mu_{R_{r_{0}}}, v_{R_{r_{0}}}\right)
\end{gathered}
$$

where the replacement was made.

$t / T_{0}=r$ is under the condition of $t=t_{r}$ Thus, process $T_{t_{0}}$ is the operating time until failure and the process $R_{r_{0}}$ of the number of failures, under the condition of (21) $r \leq r_{0}$ are mutually invertible. Their interrelation is determined by the relation (21). For a fixed $r_{0}$ the latter expression represents the distribution function of the r.v. $T_{t_{0}}$

$$
F_{T_{t_{0}}}(t)=F_{T_{t_{0}}}\left(t ; \mu_{T_{t_{0}}}, v_{T_{t_{0}}}\right), t_{0}=r_{0} \cdot T_{0}
$$

and during the operating time fixation $t_{0}$ this expression represents the distribution function of r.v. $R_{r_{0}}$

$$
F_{R_{r_{0}}}(r)=F_{R_{r_{0}}}\left(r ; \mu_{R_{r_{0}}}, v_{R_{r_{0}}}\right), r=t_{0} / T_{0}
$$

Using equalities (19) - (21), an estimate of the value of $T_{0, i}$ can be obtained for each of the distribution functions $1^{0}-6^{0}$, writing the last one for r.v. $T_{r_{c}}$ at $t=T_{R P}$ and $r_{0}=r_{i, n}^{H}$.

Let's set $\alpha_{i}=\pi_{s i}^{r e q}$, where $\pi_{s i}^{r e q}$ defined by the formula (14) and define $T_{0, i}$ as $\alpha_{i}$ - quantile of the distribution function $F_{T_{i, n}^{H}}\left(T_{R P}\right)$, i.e. solve the equation as the following:

$$
F_{r_{i, n}^{H}}\left(T_{R P}\right)=\alpha_{i}
$$

The solution of equation (24) for the exponential distribution (1) gives the followings:

$$
T_{0, i}=\frac{T_{R P}}{\mu_{r_{i, n}^{H}} \cdot \ln \left(\frac{1}{1-\alpha_{i}}\right)}
$$

For the We bull distribution (4):

$$
T_{0, i}=\frac{T_{R P} \cdot \Gamma\left(1+v_{R_{r_{i}^{H}}}\right)}{\mu_{R_{r_{i, n}^{H}}} \cdot\left[\ln \left(\frac{1}{1-\alpha_{i}}\right)\right]^{1 / v_{R}} r_{i}^{H}}
$$

While the equation (24) is being solved for a function $F_{T_{t_{0}}}(t)$ containing the function $\Phi(x)$ of a standardized normal distribution, the expansions in (20) can be used and Newton's method can be applied to the equation

$$
f(x)=0,
$$

being given by the initial approximation $x_{0}$ in the iteration procedure

$$
x_{m+1}=x_{m}-\frac{f\left(x_{m}\right)}{f \prime\left(x_{m}\right)}
$$

where $x=T_{0, i}, f(x)=F_{T_{i}^{H}}\left(T_{R P}\right)-\alpha_{i}$. As an initial approximation one of two simple estimates can be taken $\widehat{T}_{0, i}^{(1)}$ and $\widehat{T}_{0, i}^{(2)}$

$$
T_{0, i}^{(1)}=\frac{1}{n} \sum_{k=1}^{n} \frac{1}{r_{i, k}}, T_{0, i}^{(2)}=\frac{1}{n} \sum_{k=1}^{n} \frac{k}{r_{i, k}^{H}}
$$

To calculate the values of the function $\Phi(\mathrm{x})$, we use the approximation formula [11], which does not require the calculation of the density $\varphi(x)=\exp \left(-\frac{x^{2}}{2}\right)$ normalized normal distribution

$$
\Phi(x)=l-\frac{1}{9}\left(1+\sum_{i=1}^{4} c_{i} x\right)+\varepsilon(x)
$$

where

$c_{1}=0,196854, c_{2}=0,115194 ; c_{3}=0,000344, c_{4}=$ 0,019527 ;

$$
|\varepsilon(x)|<2,5 \cdot 10^{-4}
$$

and for the expansion in series of the distribution function (DN)

$$
F(t ; \mu, v)=\Phi(A)+E \Phi(B)
$$

where

$$
E=\exp \left(2 v^{-2}\right), A=\frac{t-\mu}{v \sqrt{t \mu}}, B=-\frac{t+\mu}{v \sqrt{t \mu}}
$$

We use the expansion [12]

for $A>0 F(t ; \mu, v)=1-K_{1}+E \cdot K_{2}$;

$A<0 F(t ; \mu, v)=K_{3}+E \cdot K_{2} ;(31)$

$$
\begin{aligned}
& K_{1}=\frac{1}{2}\left(1+C_{1} A+C_{2} A^{2}+C_{3} A^{3}+C_{4} A^{4}+C_{5} A^{5}+C_{6} A^{6}\right)^{-16} ; \\
& K_{2}=\frac{1}{2}\left(1-C_{1} B+C_{2} B^{2}+C_{3} B^{3}+C_{4} B^{4}+C_{5} B^{5}+C_{6} B^{6}\right)^{-16} ; \\
& K_{3}=\frac{1}{2}\left(1-C_{1} A+C_{2} A^{2}-C_{3} A^{3}+C_{4} A^{4}-C_{5} A^{5}+C_{6} A^{6}\right)^{-16} ; \\
& C_{1}=4,986734 E-02 ; C_{2}=0,021141 ; C_{3}=3,27763 E-03 ; \\
& C_{1}=3,80036 E-05 ; C_{5}=4,88906 E-05 ; C_{6}=5,383 E-06 ;
\end{aligned}
$$

In this case, for the derivative with respect to $T_{0}$ of $F(t)$, differentiating (30), we obtain

$$
F^{\prime}=\Phi^{\prime}(A) \cdot A^{\prime}+E \cdot \Phi^{\prime}(B) B^{\prime}
$$

where the prime denotes the derivative with respect to

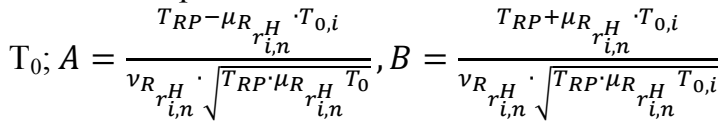
$\Phi^{\prime}(A)=\varphi(A), \Phi^{\prime}(B)=\varphi(B)$ 


$$
\begin{aligned}
A^{\prime}= & -\frac{1}{v_{R_{r_{i, n}^{H}}}} \cdot \frac{2 \cdot T_{0, i}+1}{2 T_{0, i} \cdot \sqrt{T_{R P} \cdot \mu_{R_{r_{i, n}^{H}}} \cdot T_{0, i}}}, B^{\prime} \\
& =-\frac{1}{v_{R_{r_{i, n}^{H}}}} \cdot \frac{2 \cdot T_{0, i}-1}{2 T_{0, i} \cdot \sqrt{T_{R P} \cdot \mu_{R_{r_{i, n}^{H}}} \cdot T_{0, i}}}
\end{aligned}
$$

To calculate the values of $\Gamma(\mathrm{x})$ for the gamma function, we use the well-known relation

$$
\Gamma(x+1)=x \cdot \Gamma(x)
$$

from which it follows that for $y=x+s$

$$
\Gamma(x)=\frac{\Gamma(y)}{x(x+1) \ldots \cdot(x+s-1)}
$$

Assuming that $\mathrm{s}$ is a sufficiently large natural number [13] and using the asymptotic representation [9]

$$
\Gamma(z)=\exp \left\{\left(z-\frac{1}{2}\right) \ln z-z+\frac{1}{2} \ln (2 \pi)\right\}\left[1+O\left(|z|^{-1}\right)\right],
$$

fair with $\pi-\alpha<\arg z<\pi+\alpha(\alpha-$ An arbitrarily small positive number); $O /|z|^{-1}-$ number of the order of smallness $|z|^{-1}(|z| \rightarrow \infty)$.

Expected value $a_{i}^{\text {expec }}$ waiting for the number of spare parts of the $1 \mathrm{st}$ model for the subsequent period of replenishment of spare parts SPIE:

$$
a_{i}^{\text {expec }}=T_{R P} / T_{o i}
$$

Assuming $a_{i}^{\text {expec }}$ for the initial approximation of the number of spare parts a_i, we can get a more accurate estimate for $a$ i from the condition of satisfying the required level of reliability $\pi_{\partial, i}^{R P}$ to ensure the predicted demand using the following algorithm.

Algorithm (calculation $\mathrm{a}_{\mathrm{i}}$ )

(1) $r_{i}^{H}=a_{i}^{\text {expec }}, \chi=1$

(2) If $F_{i}^{*}\left(r_{i}^{H}\right) \geq \pi_{s, i}^{r e q}$ and $r_{i}^{H} \cdot T_{0, i} \geq T_{R P}$, then go to item 4.

(3) If $\chi=2$, then go to item 8 , else go to item 5 .

(4) If $\chi=3$, then go to item 7, else go to item 6 .

(5) $r_{i}^{H}=r_{i}^{H}+1, \chi=3$ and go to item 9 .

(6) $r_{i}^{H}=r_{i}^{H}-1, \chi=2$ and go to item 9 .

(7) $a_{i}=r_{i}^{H}$ and go to item 10 .

(8) $a_{i}=r_{i}^{H}+1$ and go to item 10 .

(9) Calculate $F_{i}^{*}\left(r_{i}^{H}\right)$

(10) If $\chi \neq 1$ and go to item 2 .

(11) Print $F_{i}^{*}\left(a_{i}\right), \pi_{s, i}^{r e q}, a_{i}$.

The results of calculating $a_{i}$ from this algorithm are shown in Table 4.

Table 4. The results of the calculation of spare parts according to the proposed algorithm with initial approximation (31).

\begin{tabular}{lllll}
\hline $\boldsymbol{i}$ & $\boldsymbol{F}$ & $\boldsymbol{\pi}_{\boldsymbol{\partial}, \boldsymbol{i}}^{\boldsymbol{P}}$ & $\boldsymbol{a}_{\boldsymbol{i}}$ & $\boldsymbol{T}_{\boldsymbol{o} \boldsymbol{i}}$ \\
\hline 1 & 0,951 & 0,954 & 850 & 0,03 \\
2 & 0,962 & 0,961 & 210 & 0,18 \\
3 & 0,998 & 0,969 & 310 & 0,19 \\
\hline
\end{tabular}

\section{Conclusion}

The primary task in the calculation of spare parts for the period of replenishment of spare parts SPIA (in the Englishlanguage literature - SPIA) is the problem of identifying the distribution of applications for spare parts. Usually it is believed that the flow of applications for vehicle maintenance is Poisson and use the normal law of distribution of applications in practice, since this law is a good approximation for Poisson distribution for sufficiently large quantities of applications. However, often the requirements for Poisson flow (stationary, absence of consequences and ordinariness) can be violated, which calls into question the advisability of using the normal law of distribution of applications (for replacement of spare parts of failed parts).

In this regard, we previously solve the problem of identifying the best model for the distribution of requests for maintenance. Using Kolmogorov-Smirnov's criterion, the set of known parametric distributions (exponential, normal, lognormal, Weibull, diffusion no monotonic and monotonic) is chosen consistent with the empirical function of the distribution of applications.

Using the mutual-reversibility process $T_{t_{0}}$ of the run-tofailure distribution established in the work, with a fixed number of failures $r_{0}$ and the process $T_{r_{0}}$ for the distribution of the number of failures for a fixed running time $t_{0}$, the best model for the distribution of failures of the -th type details yields an estimate of the expected number of failures $a_{i}^{\text {expec }}$.

On the basis of these estimates, an algorithm has been developed for calculating the number of spare parts required for individual standard sizes, while fulfilling two conditions: a) the sufficiency of spare elements of type $i$ is not less than The required sufficiency index $\pi_{\partial, i}^{R P}$ of elements of a given type; b) the average time between failures of the required number of spare parts of each type is not less than the entire replenishment period $\mathrm{T}_{\mathrm{RP}}$.

The proposed methodology can be used with an arbitrary finite number of competing theoretical functions for the distribution of applications and is applicable to the calculation of spare parts in other technical branches.

\section{References}

[1] Agafonov A. V. Determination of the need for dealer car maintenance stations in spare parts and improving the efficiency of inventory management / Thesis for sisk. Scientific degree of Cand. Technical. Sciences, MADI. Moscow, 2003. - 221s.

[2] Pogrebinsky SB, Strelnikov V. P. Design and reliability of multiprocessor computers, - M.: Radio and Communication, 1988. - $168 \mathrm{p}$.

[3] Sterlingova A. N. Inventory management in supply chains Moscow Infra-M 2008.

[4] Grishin AS Development of a technique for forecasting the needs of auto service enterprises in spare parts. Thesis for socks. Scientific degree of Cand. Technical. Sciences. Moscow State Industrial University. - Moscow, 2005. - 153 p. 
[5] Maslikov A. I. Methods and models of management of multinomenclature commodity stocks in the distribution company: the dissertation... A Cand. Econ. Sci: 08.00.13/ Maslikov A. I; [Place of protection: S.-Petersburg. Polytechnical. Un-t] - St. Petersburg, 2009 - 177 p: ill. RSL OD, 61 09-8 / 3440 .

[6] Ivanov D. A. Supply chain management. St. Petersburg 2009$660 \mathrm{~s}$.

[7] Frolov Yu. N. Determination of the need for road transport in spare parts. Methodical instructions for the seminar. - MADI, Moscow, 2006. - 21c.

[8] GOST 27.005-97. Interstate standard. Reliability in technology. Models of failures. Basic provisions. -
Introduction. 01.01.99. - K.: Ed. State Standard of Ukraine, 1999. - 450s.

[9] Kulbak L. I. Basics of calculating the provision of electronic equipment with spare parts. - Moscow: Sov. radio, 1970. $186 \mathrm{~s}$.

[10] Strelnikov V. P. A new technology for investigating the reliability of machines and equipment. // Matematicheskie machine i system, 2007, No. 3, 4. - p. 228-237.

[11] Fedukhin AV, Cespedes-Garcia NV To the question of statistical reliability modeling // Mathematician machines i sistemi, 2006, №1, p. 156-163.

[12] Kuznetsov D. S. Special functions. 2 nd edition. Moscow: Higher School, 1965. - 272p. 